\title{
Cardiovascular Healthcare in 2020 - Alarming Realities in Romania
}

\author{
Roxana Hodas
}

"George Emil Palade" University of Medicine, Pharmacy, Science and Technology, Târgu Mureș, Romania

\section{CORRESPONDENCE \\ Roxana Hodas \\ Str. Gheorghe Marinescu nr. 50 540136 Târgu Mures, Romania Tel: +40 372653100 \\ E-mail: roxana.hodas@yahoo.ro}

The unprecedented progress recorded over the last decades in the field of prevention and management of cardiovascular disease (CVD) has led to a significant reduction of premature cardiovascular (CV) mortality across Europe. ${ }^{1}$ Despite this progress, a new reality is emerging and generates serious concerns for public health policies. The burden of CVD presents alarming inequalities among different European regions, remaining disproportionately larger in low- and middle-income countries (LMICs) compared to high-income countries (HICs). Moreover, economic constraints at local level involve substantial disparities in availability of $\mathrm{CV}$ care and services, with dramatic effects on the healthcare benefits of CV patients in LMICs. ${ }^{2}$

The recently published map of "ESC Cardiovascular Realities 2019", a map of ESC member countries based on monitoring CV health expenditure, infrastructure, and workforce across European countries, raised alarming signs for healthcare systems regarding the social and economic burden of CVD in different regions of Europe. ${ }^{3}$

From this point of view, Romania presents a concerning reality in all points of interest highlighted by the ESC's call to action: insufficient control of risk factors and harmful behaviors, increased burden of CVD, and disparities in availability of CV care.

Extensively investigated, the important influence of potentially reversible well-established risk factors in the determination and progression of CVD provides a strong rationale for giving a higher priority to risk reduction strategies. In HICs, the ongoing modern-day epidemics of obesity and type 2 diabetes, particularly in younger adults, represent the most serious public health challenges which threaten to erode the health gains of recent years. ${ }^{4}$

In this European context, Romania seems to present inadequate strategies. Besides the negligible decrease in hypertension prevalence, poor strategies for the identification and effective treatment of people with established hypertension contribute to the continuing high rates of myocardial infarction and CV death. Moreover, the daily intake of large quantities of alcohol, insufficient selfreported physical activity, and altered nutritional intake proved to be important 
contributors to the national burden of $\mathrm{CV}$ disease encountered in this country.

Even if longitudinal data show a steady decline of CVD mortality across European regions, mortality burden continues to show large geographical inequalities. In Romania, CVD accounts for more than $50 \%$ of all recorded deaths compared with below $30 \%$ in countries from Western Europe. In terms of CVD morbidity statistics, inequalities in CVD burden are even greater among different European countries, in correlation with national economic status. A negative association between total health expenditure per capita and age-standardized CVD burden has been identified, Romania reporting a greater than two-fold difference of disability-adjusted life years (DALYs) lost to CVD, with an average of about 9,000 DALYs per 100,000 people compared with 3,500 in HIC. ${ }^{5}$ This fact emphasizes that limited economic resources and health expenditure derive into inequitable health outcomes.

The ESC map of CVD care delivery across European countries highlights the gaps and inequalities in the availability of appropriate CV care as a consequence of the large differences in healthcare expenditure. According to recent ESC data, the Romanian healthcare system aligns with the group of countries defined by the World Health Organization as "lagging behind in infrastructure, human resource, and therapeutic procedures, mainly those with a low gross national product, typically the ESC member countries of Eastern Europe and Northern Africa". ${ }^{6}$

In terms of human resources, Romania reported 63.0 cardiologists per million people, an appalling number compared with other ESC countries such as Greece or the Republic of Georgia reporting $>250$ cardiologists per million people. This worrying situation remains the same for interventional cardiology statistics. The number of interventional cardiologists reported by Romania is 4.37 per million people, only ahead of countries such as Kyrgyzstan, Azerbaijan, or Kazakhstan, while the average number reported across ESC countries is 11.8 per million people, with an outstanding value of 30.96 per million in HICs such as Austria. The same sad reality was recorded for the density of interventional centers: 0.7 per million people in Romania compared with 6.6 per million people in Germany. The situation is even worse in the field of interventional electrophysiology, since Romania, alongside Azerbaijan and Bosnia and Herzegovina, reported less than 1 electrophysiologist per million people, while other countries, such as Poland and Sweden, have an average number of 17 electrophysiologists per million people., ${ }^{3,7}$ The situation is identical regarding the number of centers of interventional electrophysiology, ablation procedures, and device implantations. ${ }^{8}$
As at this moment we face a more than 10 -fold variation in healthcare expenditures compared to Western European countries, lagging behind in human and capital healthcare resources is readily apparent in the number of performed procedures. ${ }^{9}$ With a mean number of 4,122 coronary angiograms performed across ESC member countries, Romania reported only 1,306 procedures, much behind other countries such as Germany which reported 9,392 procedures. In terms of percutaneous coronary interventions (PCIs), only 753 procedures per million people were reported in Romania, while the average number of PCIs in Europe was estimated at 2,211 per million, and Germany reported 3,975 procedures per million people. The same disparities are recorded for structural heart interventions, Romania alongside Egypt and Turkey being placed at the bottom of the list with 25 procedures per million people, while more than 150 procedures per million are performed annually in Switzerland and Germany. Even worse, Romania is on the last place in Europe in terms of both mitral valve percutaneous interventions with 0.2 procedures per million people and transcatheter aortic valve implantations with only 2.3 procedures per million people. ${ }^{2}$

These numbers provide a stark image of the gap in human resources needed for the effective management of diagnostic and therapeutic CV procedures in Romania. Undoubtedly, much needs to be done in order to bridge the gaps in CVD healthcare delivery and to raise the quality of care in all European countries. Besides raising measures to control the well-established risk factors and harmful behavior, it is of vital importance to raise awareness, highlight inequality, advice decision-makers, and sustain investments for proper implementation of guidelines across all regions of Europe.

\section{CONFLICT OF INTEREST}

Nothing to declare.

\section{REFERENCES}

1. Vardas P, Maniadakis N, Bardinet I, Pinto F. The European Society of Cardiology Atlas of Cardiology: rationale, objectives, and methods. Eur Heart J Qual Care Clin Outcomes. 2016;2:6-15.

2. Timmis A, Townsend N, Gale C, et al. European Society of Cardiology: Cardiovascular Statistics 2017. Eur Heart J. 2018;39:508-579.

3. Timmis, A, Townsend, N, Gale, et al. European Society of Cardiology: Cardiovascular Disease Statistics 2019. Eur Heart J. 2019;1-74.

4. Capewell S, O'Flaherty M. Rapid mortality falls after risk-factors changes in population. Lancet. 2011;378:752-3

5. World Health Organisation. Global Health Observatory (GHO) data Available at: https://apps.who.int/gho/data/node.imr

6. Prüss-Üstün A, Mathers C, Corvalán C, Woodward A. Assessing the environmental burden of disease at national and local levels: Introductions 
and Methods. WHO Environmental Burden of Disease Series 1. Geneva: World Health Organisation. 2003. Available at: http://www.who.int/ quantifying_ehimpacts/publications/9241546204/en/index

7. Cenko E, Ricci B, Kedev S, et al. Reperfusion therapy for ST-elevation acute myocardial infarction in Eastern Europe: the ISACS-TC registry. Eur Heart J Qual Clin Outcomes. 2016;2:45-51.

8. Raatikainen MJ, Arnan DO, MErkely B, et al. Access to and clinical use of cardiac implantable electronic devices and interventional electrophysiological procedures in the European Society of Cardiology Countries: 2016 Report from the European Heart Rhythm Association. Europace. 2016;18:Suppl 3:iii1-iii79.

9. Walker S, Asaria M, Manca A, et al. Long-term healthcare use and costs in the patients with stable coronary artery disease: a population-based cohort using linked health records (CALIBER). Eur Heart J Qual Care Clin Outcomes. 2016;2:125-140. 